\title{
Solitary fibrous tumour along with non-small-cell lung cancer and Doege-Potter syndrome
}

\author{
Aleksandra Piórek ${ }^{1}$, Dariusz Kowalski ${ }^{1}$, Adam Płużański ${ }^{1}$, Małgorzata Szołkowska², Michał Wągrodzki ${ }^{3}$, \\ Hanna Koseła-Paterczyk ${ }^{4}$, Maciej Krzakowski ${ }^{1}$
}
${ }^{1}$ Department of Lung Cancer and Chest Tumours, Maria Skłodowska-Curie Memorial Cancer Center and Institute of Oncology, Warsaw, Poland
2Department of Pathology, Institute of Tuberculosis and Lung Diseases, Warsaw, Poland
${ }^{3}$ Department of Pathology, Maria Skłodowska-Curie Memorial Cancer Center and Institute of Oncology, Warsaw, Poland
${ }^{4}$ Department of Soft Tissue/Bone Sarcoma and Melanoma, Maria Skłodowska-Curie Memorial Cancer Center and Institute of Oncology, Warsaw, Poland

Kardiochirurgia i Torakochirurgia Polska 2019; 16 (1): 49-51

Solitary fibrous tumour (SFT) is a mesenchymal tumour usually arising from the pleura. It accounts for less than $5 \%$ of all primary pleural tumours and less than $2 \%$ of all soft tissue tumours [1]. This tumour occurs equally in both men and women and is diagnosed most frequently in the $6^{\text {th }}$ and $7^{\text {th }}$ decades of life [1]. Usually SFTs are well circumscribed (well defined) and benign. Malignant SFTs with a higher recurrence rate and distant metastatic progression are rarely seen (10-20\%) [1]. These tumours should be diagnosed by the use of combined pathologic and immunohistochemical analysis. Malignant tumours present with increased pleomorphism and cellularity, increased mitotic activity (> 4 mitoses per high power field), extensive necrosis and haemorrhage. In more than $50 \%$ of patients the disease is asymptomatic and pulmonary lesions are diagnosed incidentally during follow-up examinations. The remaining patients usually present with dyspnoea and chest pain. Paraneoplastic syndromes are rarely seen. The most commonly reported paraneoplastic syndromes are PierreMarie-Bamberg syndrome and Doege-Potter syndrome, which is characterized by a large tumour with increased excretion of insulin-like growth factor 2 (IGF-2) causing hypoglycaemia [1].

A 69-year old woman with the diagnosis of non-smallcell lung cancer (NSCLC) of the right lung was admitted to the hospital in May 2009. Chest computed tomography (CT) revealed a well-circumscribed, polycyclic lesion in the frontal part of the left lung apex and irregular infiltration $(20 \mathrm{~mm}$ diameter) in the $2^{\text {nd }}$ segment of the right lung. No other lesions within both lungs were detected. The examination did not reveal enlarged lymph nodes or presence of fluid in the pleural cavity. Bronchofibroscopy did not reveal intrabronchial changes. Fine-needle biopsy of the left lung apex lesion was performed, but it was non-diagnostic. The biopsy of the right lung lesion was performed and adenocarcinoma was diagnosed. Positron emission tomography-CT (PET-CT) was performed to assess the clinical stage of the disease. The result indicated an active, metabolic proliferative process in the right lung (nodule of $16 \times 24 \mathrm{~mm}$ in size, 18-fluorodeoxyglucose maximum standardized uptake value -

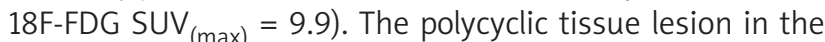
left lung apex $(36 \times 36 \times 30 \mathrm{~mm})$, adjacent to the pleura, did not accumulate 18F-FDG (18F-FDG SUV $(\max )=1.0)$. No lymph node enlargement or increased 18F-FDG accumulation was found and the disease was classified as CT1bNOMO - CS IA. The general condition was good (Karnofsky Performance Score - KPS of 90 corresponding to WHO score of 1). Medical history revealed nicotine addiction, hypertension and tuberculosis in her youth. No clinically relevant abnormalities in physical examination and laboratory results were found. Right upper lobectomy plus lymphadenectomy was performed in August 2009. The postoperative pathology report confirmed NSCLC of the right lung with stage pT1bNOMORO and no adjuvant treatment was used. The patient was monitored after surgery. Follow-up imaging did not reveal any recurrent changes in the right lung, but slow enlargement of the left lung lesion was observed since 2010. The patient refused invasive diagnostic procedures for the left lung lesion and she was monitored due to lack of clinical symptoms. Chest CT done in 2012 revealed a tumour $50 \times 108 \mathrm{~mm}$ in diameter (Fig. 1). The tumour caused pain in the chest wall and the patient agreed to the biopsy procedure. In February 2013 core needle biopsy of the tumour in the left lung was performed. The pathology report revealed spindlecell carcinoma. No mitotic activity or necrosis was found. The microscopic image and immunohistochemical profile indicated type 1 SFT. The patient refused a surgical procedure and remained monitored afterwards. Imaging revealed fur-

Address for correspondence: Aleksandra Piórek, Department of Lung Cancer and Chest Tumours, Maria Skłodowska-Curie Memorial Cancer Center and Institute of Oncology, 5 Roentgena St, 02-781 Warsaw, Poland, e-mail: opiorek@tlen.pl

Received: 25.11.2018, accepted: 15.01.2019. 

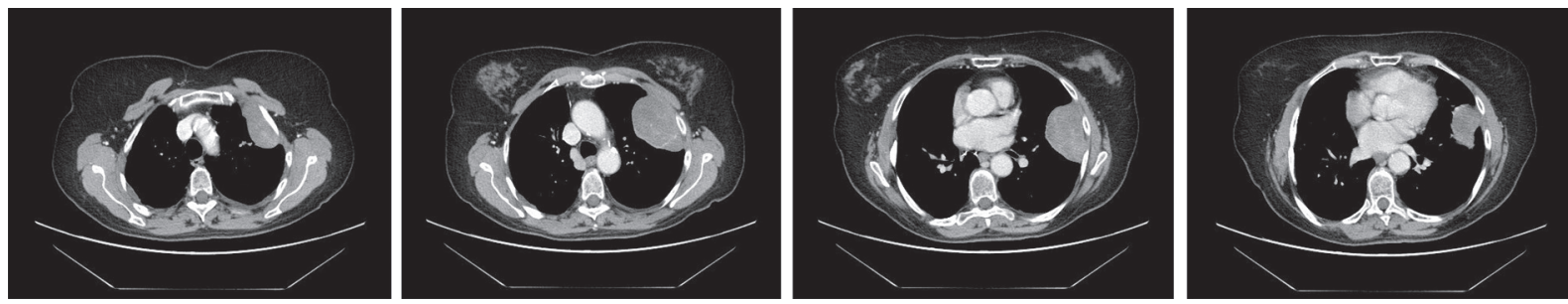

Fig. 1. Computed tomography scans of the thorax. Large polycyclic pleural tumour from the level of the $2^{\text {nd }}$ to $6^{\text {th }}$ rib
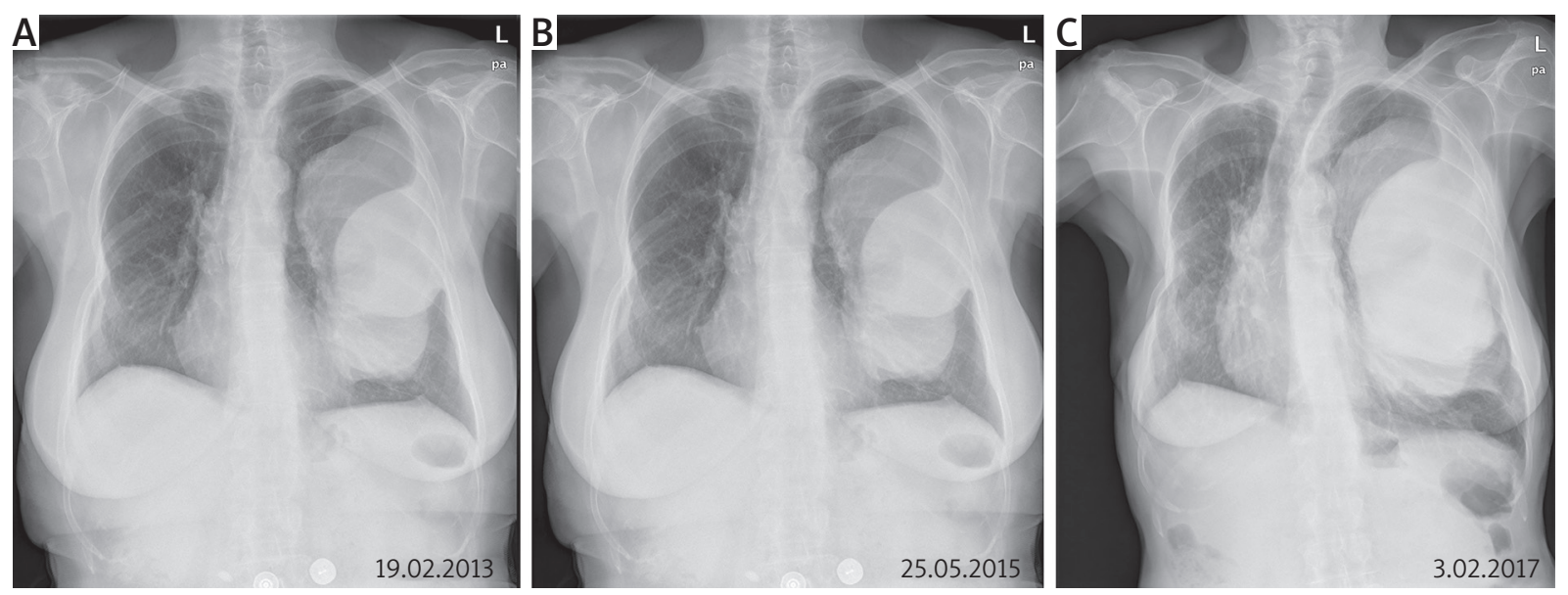

Fig. 2. Posteroanterior chest radiograph (A-C). Mass located in left lung. Posteroanterior chest radiograph completed at yearly intervals from 2013 through 2017 (images from 2014 and 2016 not shown)

ther radiological progression (Fig. 2). The patient was hospitalized in the endocrinology department in March 2017, because of recurrent episodes of hypoglycaemia in the last 2 months (lowest value $\left.-20 \mathrm{mg} \%, \mathrm{HbA}_{1 \mathrm{c}}-4.5 \%\right)$. The symptoms disappeared after $5 \%$ glucose administration. Insulinoma diagnosis was initially considered due to focal change found in the pancreatic tail on abdominal CT $(12 \times 12 \mathrm{~mm})$, which was not confirmed during further examinations. Subsequently hypoglycaemia episodes were linked to the Doege-Potter syndrome. Hydrocortisone was administered. After completion of diagnostics the patient agreed to undergo a surgical procedure. Resection of the pleural tumour on the left side was performed on April 2017. The pathology report revealed two neoplastic foci of $20 \mathrm{~cm}$ and $14 \mathrm{~cm}$ in diameter. Mitotic activity was 2-5/10 HPF with focal necrosis. Microscopic imaging indicated malignant fibrous tumour of the pleura (type 2) (Fig. 3). Both lesions were removed (surgical margin below $0.1 \mathrm{~cm}$ ). Hypoglycaemia episodes did not recur after surgery. Chest CT in May 2017 showed no signs of recurrence. The patient continues follow-up.

The development of improved diagnostic techniques contributed to the higher frequency of multiple primary malignant neoplasms (MPMN). The MPMN can be synchronous or metachronous. To the best of our knowledge, this is the second report of a patient presenting with these two pathological entities simultaneously. The first one was a 57-year-old woman with comorbid malignant solitary fibrous tumour of the pleura and adenocarcinoma of the lung. The patient was treated successfully for both diseases via a one-stage operation through median sternotomy with good results [2]. In the present case we observed two lesions (one in the left lung and another in the right lung). The right lung lesion was diagnosed as NSCLC and it was successfully resected. Negative results of the first biopsy and PET-CT along with malignant primary lung cancer probably caused a lack of diagnostic continuity and progression of the left lung tumour, neoplastic transformation and presence of life-threatening hypoglycaemia episodes.

Solitary fibrous tumour in the chest CT usually presents as a homogeneous, well-circumscribed soft tissue mass adjacent to the pleural surface. However, CT imaging diagnostic accuracy is not high. It is caused by the low number of patients and diversified SFT image depending on size and location (chest wall, lungs, diaphragm, mediastinum) [3]. Diagnostic accuracy of imaging was $56.1 \%$ in one study and the SFT diagnosis was not considered in the differential diagnosis in $25 \%$ of patients [3]. In 4 patients with a benign solitary fibrous tumour of the pleura (SFTP) the mean maximum SUV (maxSUV) on PET-CT was 2.0 (1.2-3.1) and in 5 patients with malignant SFTP it was $3.6(2.5-4.9)$ $(p=0.049)$ [3].

Hypoglycaemia - the main sign of insulinoma - may also be observed in other cancers and then it is called non-islet cell tumour hypoglycaemia (NICTH). In SFT cases, especially located in the pleura or mediastinum, the hypoglycaemia is called Doege-Potter syndrome [5]. DoegePotter syndrome is the second most frequent after PierreMarie-Bamberg paraneoplastic syndrome associated with SFT and its incidence is approximately 5\% (4-14\%) [1, 4]. The presence of hypoglycaemia does not correlate with 

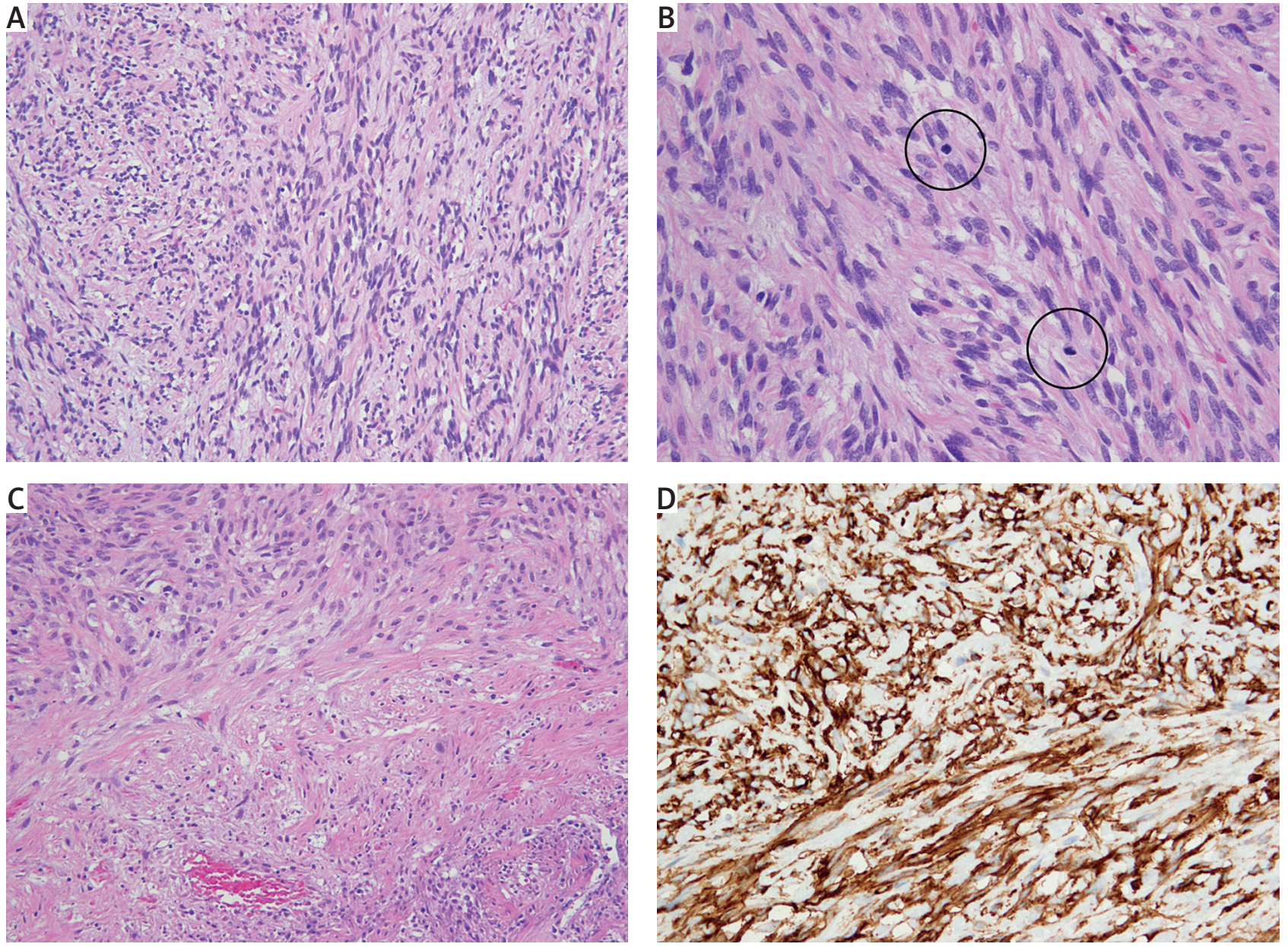

Fig. 3. Histopathology of malignant solitary fibrous tumour of the pleura (A-C). On medium power spindle-shaped tumour cells of limited cytological atypia forming long fascicles of medium to high cellularity were seen (A). Mitotic figures (B, circles) were numerous (5/10 high power fields) and there were widespread areas of necrosis (C, Nec). D - All neoplastic cells showed strong expression of CD34 (A, B, C haematoxylin and eosin staining, D - anti-CD34 immunohistochemical reaction; A, C, D - magn. 200×, B - magn. 400×)

the size or clinical stage of neoplastic process, although diagnosed tumours are usually large. The average size of the reported tumours resulting in hypoglycaemia is $20 \mathrm{~cm}$ (54\% of benign tumours and $42 \%$ of malignant tumours) [4]. Increased insulin growth factor-2 (IGF-2) excretion is considered as the main cause of hypoglycaemia, especially in cases of excretion of its incomplete form ("big" IGF-2) [4-6]. The NICTH patients should avoid prolonged fasting. Regular meals are recommended, including meals during the night. In case of emergency intravenous administration of glucose or subcutaneous administration of glucagon should be considered [5]. Glucocorticosteroids (especially hydrocortisone and prednisolone) are usually administered to stabilize the patient's condition until the location of the neoplastic lesion and further treatment schedule are established [5]. The treatment of the underlying disease is essential within the management of NICTH. Solitary fibrous tumour is treated by surgical removal [1]. In malignant SFTs a wide surgical margin should be obtained to avoid cancer recurrence. The recurrence rate in malignant SFT is 14-68\% in 2 years of follow-up (even after radical resection) $[1,4]$. At present there are no adjuvant therapy or follow-up rec- ommendations in patients with resected SFTP. Imaging every 6 months in the first 2 years after the diagnosis and then once a year has been reported in the literature [1].

\section{Disclosure}

The authors report no conflict of interest.

\section{References}

1. Chick JF, Chauhan NR, Madan R. Solitary fibrous tumors of the thorax: nomenclature, epidemiology, radiologic and pathologic findings, differential diagnoses, and management. AJR Am J Roentgenol 2013; 200: 238-248.

2. Watanabe S, Nakamura Y, Sakasegawa K, Kariatsumari K, Yotsumoto D, Sakata R, Gezima K. Synchronous solitary fibrous tumor of the pleura and lung cancer. Anticancer Res 2003; 23: 2881-2883.

3. Yeom YK, Kim MY, Lee HJ, Kim SS. Solitary fibrous tumors of the pleura of the thorax: CT and FDG PET characteristics in a tertiary referral center. Medicine (Baltimore) 2015; 94: e1548.

4. Kalebi AY, Hale MJ, Wong ML, Hoffman T, Murray J. Surgically cured hypogly cemia secondary to pleural solitary fibrous tumour: case report and update review on the Doege-Potter syndrome. J Cardiothorac Surg 2009; 4: 45.

5. Krysiak R, Okopień B. Paraneoplastic endocrine syndromes. Przegl Lek 2016; 73: 29-39.

6. Potempa M, Jonczyk P, Zalewska-Ziob M. Endocrinological view on lung cancer. Onkol Prak Klin 2015; 11: 121-128. 